\title{
Educação de pacientes com asma: atuação do enfermeiro*
}

\author{
Ana Rita de CÁssia Bettencourt ${ }^{1}$, Maria Alenita de Oliveira², \\ ANA LUISA GOdOY FERNANDES ${ }^{3}$, Miguel BOGOSSIAN ${ }^{4}$
}

\begin{abstract}
Introdução: Programas de educação em asma levam a melhor controle da asma e são uma das recomendações dos consensos. Os programas devem incluir uma enfermeira no grupo educacional que aplicará o programa estruturado. A proposta da intervenção é mudar a vida diária do paciente e sua família e melhorar o controle da doença e qualidade de vida. Objetivo: Padronizar e aplicar um

modelo estruturado de pós-consulta, como parte de atendimento multidisciplinar dentro de um

programa educativo para pacientes asmáticos em um ambulatório de um hospital público e acompanhamento das mudanças no conhecimento da asma e qualidade de vida. Método: Estudo longitudinal, prospectivo, com duração de seis meses, realizado no ambulatório de Pneumologia do Hospital São Paulo (Unifesp/EPM), dentro de um programa de educação para pacientes asmáticos, em que foram agendadas seis consultas, com intervalo de quatro semanas, com os seguintes temas: o que é asma, medicações de alívio e prevenção, diário de sintomas e escore de dispnéia, uso correto do aerossol, discussão dos fatores desencadeantes e profilaxia, e também reconhecer os sinais de descontrole da asma. Um modelo padronizado do questionário de conhecimentos sobre asma e outro sobre qualidade de vida foram aplicados no início, durante e no final do estudo. Resultados: 26 asmáticos foram acompanhados por seis meses e mostraram melhora significante na identificação dos problemas relacionados ao manejo da asma, como também nos índices de qualidade de vida.

Conclusão: $O$ modelo de atendimento, como também as técnicas de treinamento, foram adequadas e úteis no desenvolvimento de um programa educativo estruturado para pacientes asmáticos.
\end{abstract}

(J Pneumol 2002;28(4):193-200)

\section{Education of the asthmatic patient: the nursing approach}

Background: Asthma education programs induce better asthma control and are one of main recommendations in guidelines. The programs recommendations should include a nurse in the educational team applying the structured program. The purpose of the intervention is to bring a change in the daily life of the patient and the family in order to improve disease control and quality of life. Aim: To standardize and apply a structured post-consultation model as part of multidisciplinary care in an educational program for asthmatic patients seen at the outpatient clinic of a public hospital, and to monitor the changes in asthma knowledge and quality of life.

Methods: A longitudinal prospective study over six months with regular visits at four week intervals (total $=6$ visits), conducted at the outpatient department of Unifesp/EPM/HSP, and the patients were included after signing a consent form. The asthma structured educational plan was conducted by a multidisciplinary team and addressed the following issues: what is asthma, relief and prevention medicines, daily report of symptoms and dyspnea score, training of the correct use of inhaled medication, discussion and actions regarding triggering factors and how to avoid them, and tips to recognize the signals of uncontrolled asthma. A standard questionnaire about general knowledge on asthma and a quality of life questionnaire were applied at the beginning, during the course, and at the end of the program. Results: 26 asthmatics were followed over six

* Trabalho realizado na Universidade Federal de São Paulo.

1. Enfermeira; Doutora em Ciências Pneumológicas.

2. Doutora em Pneumologia.

3. Professora Adjunta da Disciplina de Pneumologia.

4. Professor Titular e Chefe da Disciplina de Pneumologia da Faculdade de Medicina Unisa.
Endereço para correspondência - Ana Rita de Cássia Bettencourt, Rua Botucatu, 1.059, casa 5 - 04023-062 - São Paulo, SP. Tel. 55493813; e-mail: anarita@denf.epm.br

Recebido para publicação em 11/12/01. Aprovado, após revisão, em 3/6/02. 
months and showed a statistically significant improvement in their identification and management of asthma skills as well as better quality of life indices. Conclusion: The model of care and the approach and training techniques were adequate and useful in the development of a structured educational program for socially deprived patients.

Descritores - Asma. Enfermagem. Educação. Qualidade de vida.

Key words - Asthma. Nursing. Education. Quality of life.

\section{INTRODUÇÃO}

A asma é uma doença crônica que afeta cerca de $10 \%$ da população, sendo considerada um sério problema de saúde pública. Nos Estados Unidos, a asma afeta cerca de 14 a 15 milhões de pessoas, sendo responsável por 470 mil hospitalizações e cinco mil mortes/ano ${ }^{(1)}$. Em termos econômicos, estima-se um gasto anual de 6,4 bilhões em custos diretos e indiretos, sendo que há correlação entre a gravidade e o custo econômico da asma(2).

No Brasil, a asma foi a 4⿳亠丷⿵冂⿱十口𧰨 causa de internação (1996) nos hospitais do Sistema Único de Saúde e é responsável por uma média de 2.000 óbitos/ano, aproximadamente $70 \%$ destes durante a hospitalização dos doentes ${ }^{(3)}$.

Vários autores demonstraram que o aumento da mortalidade e morbidade observado na doença está associado, entre outros fatores, a abordagem inadequada da asma pelos médicos e pacientes ${ }^{(4-6)}$.

A asma é uma doença de tratamento complexo que exige as participações ativas de seus portadores e familiares e leva a limitações físicas, emocionais e sociais. Para seu controle, além do tratamento farmacológico adequado, é necessário que o doente tenha noções sobre a asma, quais os fatores desencadeantes e como evitá-los, e adquira habilidades como o uso correto das medicações $e$ reconhecer os sinais de controle e descontrole da doença ${ }^{(7)}$.

Com base nesse conhecimento, vários programas de educação foram aplicados, demonstrando redução dos parâmetros de morbidade da asma, com diminuição do número de visitas ao pronto-socorro e de hospitalizações, redução dos sintomas e melhora da qualidade de vida ${ }^{(8-10)}$. $\mathrm{O}$ nosso grupo ambulatorial de asma demonstrou que a aplicação de um programa de educação em nosso meio também resulta em redução dos índices de morbidade e melhora de qualidade vida ${ }^{(11,12)}$.

A educação do paciente asmático, portanto, é um dos pilares do tratamento da asma, conforme preconizado pelo GINA ${ }^{(13)}$.

Os programas de educação são baseados nas orientações recomendadas nos consensos e devem ser aplicados associados ao atendimento médico, sendo adaptados às características socioeconômico-culturais da população-

\author{
Siglas e abreviaturas utilizadas neste trabalho \\ C1 - Primeira consulta \\ C2 - Segunda consulta \\ C3 - Terceira consulta \\ C4 - Quarta consulta \\ C5 - Quinta consulta \\ C6 - Sexta consulta \\ GINA - Global initiative for asthma \\ PFE - Pico de fluxo expiratório
}

alvo. A sua condução é multidisciplinar e pode ser realizada por médicos, fisioterapeutas e enfermeiros ${ }^{(14)}$.

O papel da enfermagem em prol da saúde coletiva é reconhecido; o enfermeiro atua como mediador entre a comunidade e o sistema de saúde local, tendo como objetivo a educação em saúde. A atuação da enfermagem é descrita em vários programas de educação em asma, tendo a responsabilidade de educar os pacientes e sua família ${ }^{(15,16)}$.

O objetivo deste trabalho foi desenvolver um modelo de pós-consulta padronizado para avaliar o aprendizado e acompanhar a evolução clínica do paciente durante o programa de educação. Esta pós-consulta, denominada consulta de enfermagem, foi realizada pela enfermeira que fazia parte do grupo ambulatorial de asma.

\section{MÉTODO}

Tendo em vista a importância da transmissão de conhecimento, treinamento $e$ necessidade de reforçar $e$ apoiar as diversas etapas do tratamento do asmático, desenvolvemos as seguintes etapas:

1) Aplicar e avaliar um questionário criado especificamente para revisar e reforçar o conhecimento sobre asma.

2) Acompanhar ao longo do tempo a transmissão do conhecimento, ou seja, o grau inicial e de aprendizado em relação a: o que é asma, medicações de alívio e prevenção, identificação dos fatores desencadeantes, medidas de profilaxia ambiental, sinais de controle e descontrole da asma, preenchimento do diário e técnica de uso correto do aerossol.

3) Avaliar globalmente o programa através da aplicação de questionário de qualidade de vida.

O estudo foi longitudinal, prospectivo, com duração de seis meses, dentro de um programa de atendimento ambulatorial educativo, em que cada paciente foi seu próprio controle, sendo obtido o consentimento por escrito para participação. As consultas eram agendadas com in- 
tervalo de quatro semanas, em número total de seis ( $\mathrm{C} 1 \mathrm{a}$ C6). Os pacientes tinham diagnóstico confirmado de asma segundo os critérios da American Thoracic Society $(\mathrm{ATS})^{(17)}$ e foram selecionados de forma aleatória através do cadastro computadorizado do ambulatório de asma da Disciplina de Pneumologia da Universidade Federal de São Paulo (Unifesp), independente da gravidade da expressão da doença e/ou adesão ao tratamento. As características dos pacientes incluídos no estudo estão apresentadas na Tabela 1. Não foi fornecido nenhum tipo de medicamento; a única intervenção ativa foi o programa educativo. Em cada consulta o programa de educação foi associado ao atendimento médico; a medicação era ajustada conforme a necessidade clínica, com base nas recomendações do GINA $\left.{ }^{(13)}\right)$. No Quadro 1 apresentamos o plano de atendimento ambulatorial para aplicação do programa de educação estruturado, que era realizado por vários profissionais da área da saúde, médicos e não médicos.

Para acompanhamento da efetividade da aplicação da pós-consulta foi utilizado um cronograma baseado na experiência prévia da investigadora, atuando como observadora de um grupo de pacientes participantes de um programa de educação em asma implantado no ambulatório da instituição. Esse instrumento foi composto de quatro partes:

a) Características demográficas;

b) Levantamento das condições ambientais e pessoais;

c) Identificação dos problemas relacionados à asma;

d) Avaliação de aquisição de conhecimento durante o programa;

A abordagem dos assuntos durante as consultas do programa foi assim distribuída (Anexo 1):

Pós-consulta 1: O que é asma; medicação de alívio ou broncodilatador, e de prevenção ou antiinflamatória. Entrega do folheto explicativo e registro diário.

TABELA 1

Características clínicas e socioeconômicas dos pacientes que participaram do estudo

\begin{tabular}{llr}
\hline Sexo $(\mathrm{n})$ & Masculino & 3 \\
& Feminino & 19 \\
\hline Classificação clínica $(\%)$ & Moderada & 50 \\
& Grave & 50 \\
\hline Idade $(\bar{x})$ & & 37 \\
\hline Escolaridade $(\%$ doentes) & Analfabetos & 14 \\
& $<5$ anos & 68 \\
& $>5$ anos & 18 \\
\hline Renda mensal (\% doentes) & Até 5 sal. mínimos* & 82 \\
& $>5$ sal. mínimos & 18 \\
\hline
\end{tabular}

* Salário mínimo vigente: $\mathrm{R} \$ 120,00$

J Pneumol 28(4) - jul-ago de 2002
Pós-consulta 2: Fatores desencadeantes e a importância da profilaxia. Orientar o paciente para trazer as embalagens dos medicamentos que usa para asma na próxima consulta.

Pós-consulta 3: Pedir para o paciente identificar e separar as embalagens de medicação de alívio e prevenção. Orientar o paciente para chegar 40 minutos antes no próximo retorno para assistir a uma fita de vídeo de reforço sobre o que é asma, medicação de alívio e prevenção e técnica correta do uso de aerossol.

Pós-consulta 4: Explicar sinais de controle e descontrole da asma. Perguntar o que entendeu da aula (fita de vídeo) apresentada na pré-consulta e esclarecer as dúvidas. Orientar para chegar 40 minutos antes no próximo retorno para assistir à fita de vídeo de reforço sobre sinais de controle e descontrole da asma.

Pós-consulta 5: Perguntar o que entendeu da aula (fita de vídeo) apresentada na pré-consulta e esclarecer as dúvidas.

Pós-consulta 6: Informar ao paciente o término do programa e seu aproveitamento (avaliação do programa).

Os seguintes itens eram comuns a todas as consultas:

a) Identificar problemas e propor intervenções de controle e erradicação;

b) Questionar sobre as informações que recebeu na consulta anterior e esclarecer as dúvidas relatadas pelo paciente;

c) Verificar o preenchimento do registro diário;

d) Checar e/ou ensinar a técnica de uso correto do aerossol;

\begin{tabular}{|l|}
\hline \multicolumn{1}{|c|}{ QUADRO 1} \\
Etapas de atendimento do programa ambulatorial \\
\hline 1) Pré-consulta \\
- Medida do PFE pré e 15 minutos após o uso do broncodila- \\
tador; \\
- Verificação da pressão arterial, pulso e freqüência respira- \\
tória; \\
- Avaliação da técnica de uso do aerossol. \\
2) Consulta médica \\
- Exame físico, diário de sintomas, medicações em uso e efei- \\
tos colaterais; \\
- Receita médica e plano de ação. \\
3) Pós-consulta = consulta de enfermagem \\
- Características demográficas; \\
- Levantamento das condições ambientais e pessoais; \\
- Identificação dos problemas relacionados à doença e inter- \\
- - Avançõasiação do conhecimento e habilidades do paciente a \\
partir das informações recebidas no decorrer do plano de \\
educação. \\
4) Aulas e discussões de 30 minutos, em grupo, agendadas após \\
o atendimento ambulatorial.
\end{tabular}


e) Anotar e explicar a receita médica;

f) Entregar e explicar o registro diário.

Os itens abordados foram explicados através de discussões em grupos, em duas ocasiões. Em cada pós-consulta as orientações sobre conceito e fisiopatologia foram feitas com linguagem simples, através de cartazes com informações visuais para facilitar o aprendizado.

A avaliação da aprendizagem era feita em todas as consultas através de questionário padronizado contendo os tópicos abordados no programa.

2) Questionário de qualidade de vida

O questionário de qualidade de vida adaptado ao nosso meio socioeconômico-cultural foi aplicado no início e ao término do programa e abrangeu cinco áreas: limitação das atividades físicas preferenciais do paciente; freqüência e gravidade dos sintomas; adesão ao tratamento; nível socioeconômico, e aspectos psicossociais, conforme técnica descrita anteriormente pelo nosso grupo ${ }^{(18)}$.

3) Da escala utilizada para avaliação do uso do aerossol

A escala utilizada para avaliação do uso do aerossol foi baseada na de Manzella et al.(19), conforme as etapas descritas:

1) Agitar a aerossol por vários segundos;

2) Exalar profundamente antes da inalação;

3) Inclinar o pescoço para trás antes da inalação;

4) Segurar o aerossol na posição correta (verticalmente, com o bocal para baixo na direção da boca);

5) Se usar espaçador, manter os lábios fechados e o tubo entre os dentes durante o jato; se não usar espaçador, o aerossol deve estar a uma distância de $2,5 \mathrm{~cm}$ da boca durante o jato;

6) Inspirar lenta e profundamente após o jato;

7) Segurar a respiração por dez segundos após a inalação;

8) Esperar um minuto antes da próxima dose.

Para avaliar o uso do aerossol foi considerado o seguinte escore: uso correto - oito passos certos; incorreto - menos de oito passos certos.
4) Registro diário

O registro diário utilizado neste programa de educação foi um registro simples, no qual o doente anotava a ausência ou presença de sintomas durante o dia e durante a noite. Também foi anotada a quantidade de broncodilatador utilizada. Caso o paciente não soubesse ler, um parente era solicitado para anotar os dados.

\section{Análise estatística}

Para analisarmos os dados coletados foi utilizado o teste $\mathrm{G}$ de Cochran para análise das percentagens de acerto dos itens abordados nas consultas de enfermagem de $\mathrm{C} 1$ a C6 e o teste de Wilcoxon para análise dos índices do questionário de qualidade de vida no início e após o programa $^{(20)}$.

\section{REsUltados}

Foram incluídos 26 portadores de asma brônquica; destes, quatro foram excluídos por perda de seguimento. As características dos pacientes incluídos no protocolo estão listadas na Tabela 1.

1) Da aplicação do questionário

A sua aplicação levava em torno de 15 minutos e não foi observada dificuldade para responder às perguntas formuladas.

2) Avaliação do conhecimento e do aprendizado

Na Tabela 2, apresentamos a avaliação do conhecimento e do aprendizado dos pacientes durante o programa de educação. Na $\mathrm{C} 1$ nenhum dos pacientes sabia dizer "o que é asma". Na C2, somente 50\% dos doentes sabiam o que era a doença, sendo necessária mais uma sessão educativa para que $90 \%$ dos doentes soubessem defini-la adequadamente.

Da mesma maneira, podemos observar que, para medicação de alívio, os pacientes passaram a adquirir o conhecimento a partir da C3.

$\mathrm{O}$ índice de acerto dos pacientes quando questionados sobre os fatores desencadeantes já foi estatisticamente

TABELA 2

Avaliação do conhecimento adquirido dos doentes com asma durante o programa de educação: percentual de acertos nas questões avaliadas

\begin{tabular}{lccccccc}
\hline \multirow{2}{*}{ Questões formuladas } & \multicolumn{5}{c}{ \% de acertos no decorrer das consultas } \\
\cline { 2 - 7 } & C1 & C2 & C3 & C4 & C5 & C6 \\
\hline O que é asma? & 0 & 50 & $90,9 *$ & 100 & 95,5 & 86,4 \\
Medicações de alívio & 22,7 & 50 & $95,5^{*}$ & 100 & 100 & 90,9 \\
Medicações de prevenção & 22,7 & 50 & $90,9 *$ & 100 & 100 & 90,9 \\
Identificação dos fatores desencadeantes & $54,5^{*}$ & 54,5 & 90,9 & 81,8 & 95,5 & 100 \\
Medidas de profilaxia ambiental & 22,7 & 27,3 & $86,4^{*}$ & 90,9 & 95,5 & 95,5 \\
Sinais de controle e descontrole da asma & 0 & 0 & 0 & 9,1 & 77,3 & $95,5^{*}$ \\
\hline$*$ \% de acertos significante $(p<0,05)$ & & & & & &
\end{tabular}


TABELA 3

Avaliação das habilidades adquiridas pelos doentes durante o programa de educação: percentual de acertos no decorrer das consultas

\begin{tabular}{lccccccc}
\hline \multirow{2}{*}{ Habilidades } & \multicolumn{5}{c}{$\begin{array}{c}\text { \% de acertos no } \\
\text { decorrer das consultas }\end{array}$} \\
\cline { 2 - 7 } & C1 & C2 & C3 & C4 & C5 & C6 \\
\hline Preenchimento do diário & 0 & $59,1 *$ & 72,7 & 86,4 & 86,4 & 90,4 \\
Uso correto do aerossol & $36,4^{*}$ & 59,1 & 63,6 & 72,7 & 81,8 & 86,4 \\
\hline$* \%$ de acertos significante $(p<0,05)$ & & & & & &
\end{tabular}

significante na C1 (54,5\%), em contraste com o índice de acerto de profilaxia ambiental, que foi de $22,7 \%$, tendo aumento significante somente após a C3.

Em relação ao tema "sinais de controle e descontrole da doença", observamos que nas três primeiras consultas o índice de acerto foi nulo, fato esperado, já que este item só foi explicado na C4. Somente 9\% dos doentes sabiam definir sinais de controle e descontrole da doença antes da abordagem do tema, tendo aumento significante após a sessão educativa.

3) Avaliação das habilidades adquiridas durante o programa de educação

Na Tabela 3, encontramos o percentual de acertos dos doentes em relação às habilidades adquiridas durante o estudo. Em relação ao preenchimento do registro diário, observamos que o índice de acerto na C2 foi de 59,1\%, aumentando progressivamente, porém não alcançando $100 \%$ de acerto, mesmo com o reforço em todas as consultas.

$\mathrm{Na} \mathrm{C} 1,36,4 \%$ dos doentes sabiam utilizar adequadamente o aerossol; este índice foi aumentando no decorrer do programa de educação, porém o máximo de acerto encontrado foi de $86,4 \%$ na última consulta.

4) Impacto do aprendizado na qualidade de vida

Na Figura 1, temos os escores de qualidade de vida obtidos pelos pacientes antes e após o programa de educação e observamos que houve redução significante do percentual de limitação dada pela asma na qualidade de vida global e nas áreas limitação física, freqüência e gravidade, adesão e socioeconômico. Não observamos diferença dos escores da área psicossocial antes e após a aplicação do programa.

\section{DISCUSSÃO}

A conscientização e a educação são fatores fundamentais para um adequado controle da doença, possibilitando melhor convívio com esta e tornando o indivíduo capaz de prevenir e detectar as principais complicações ${ }^{(21)}$.

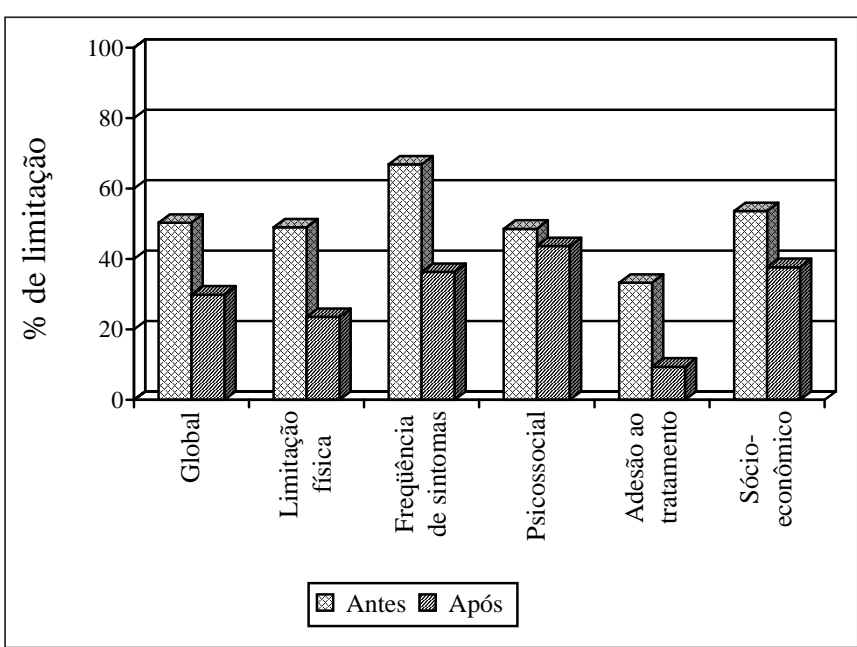

Figura 1 - Escores de qualidade de vida dos pacientes antes e após o programa de educação

Observamos que a aplicação do questionário elaborado foi factível, levando à racionalização do atendimento do asmático, sendo mais fácil a identificação dos problemas relacionados ao manejo do paciente.

No decorrer das seis consultas de enfermagem, oito itens foram abordados e reforçados a cada consulta. Observamos melhora significante de todos os parâmetros relacionados ao aprendizado. A pergunta o que é asma? foi feita no início da primeira consulta e constatamos que nenhum dos pacientes soube conceituá-la, sendo necessária a orientação sobre as características crônicas da doença. Além de ser a base para o entendimento dos itens seguintes, o esclarecimento possibilita o estabelecimento de um elo entre a enfermeira e o paciente, favorecendo a adesão ao programa proposto $e$ ao tratamento (22).

A técnica correta do uso do aerossol é fundamental para que a medicação seja efetiva. Para poder ensinar a técnica correta, a equipe deve ser treinada até estar apta. Rebuck et al. ${ }^{(23)}$ verificaram que os médicos têm mostrado, freqüentemente, pobre conhecimento sobre o uso correto do aerossol, e que uma breve aula é suficiente para produzir aumento significativo no conhecimento $e$ habilidade para utilizá-la corretamente. Sandrini et al. ${ }^{(24)}$ demonstraram em seu estudo que a maioria dos pacientes aprende a usar corretamente, ou quase corretamente, a medicação em aerossol após uma primeira explicação, desde que exaustivamente ensinada.

No nosso estudo efetuamos a demonstração da técnica e correção passo a passo. Para a utilização correta desse dispositivo, muitos aspectos devem ser levados em consideração, tais como: coordenação dos movimentos inspiratórios e expiratórios, coordenação motora, destreza 
manual para acionar o dispositivo, utilização seqüencial e correta das etapas do uso da medicação inalatória. Acreditamos que, por esse motivo, somente $36,4 \%$ utilizavam corretamente o aerossol na $\mathrm{C} 1$, conforme a Tabela 3 .

Para manter a asma sob controle é fundamental que os fatores desencadeantes sejam identificados e que as medidas de profilaxia sejam realizadas. Esse item foi abordado no decorrer da C2. Observamos que apesar de esse tema ainda não ter sido explicado, na C1 e C2, 54,5\% dos pacientes reconheciam os fatores que provocavam a exacerbação da doença. Mesmo reconhecendo, somente $22,7 \%$ dos pacientes faziam corretamente a profilaxia desses fatores desencadeantes na $\mathrm{C} 1$, o que reforça a importância de orientação adequada.

Existe diferença entre o reconhecimento dos problemas pelo paciente e a mudança de comportamento em relação à doença, principalmente em termos de ações profiláticas e de condutas a serem seguidas durante a crise de asma ${ }^{(25)}$. Fatores psicológicos e socioeconômicos têm grande influência sobre o conhecimento e comportamento ${ }^{(26)}$. Existe necessidade de trabalharmos individualmente com essas dificuldades para obtermos resultados satisfatórios.

Os sinais de controle e descontrole da doença foram abordados a partir da C3. A opção para abordar os itens de forma progressiva se deve ao fato de que no processo de aprendizado é necessário que o paciente já tenha alguns conhecimentos para o entendimento adequado de uma nova informação(27). Mesmo com todas as informações recebidas durante o programa de educação, somente $9 \%$ dos pacientes definiram adequadamente o que seriam sinais de descontrole e controle da doença, quando abordado inicialmente o tema na $\mathrm{C} 4$. A dificuldade de reconhecimento de exacerbação é um dos fatores limitantes para o adequado manejo da asma. Segundo Osman ${ }^{(28)}$, a educação em asma baseada somente em informações gerais não é efetiva para prover o autotratamento; os pacientes devem saber como controlar seus sintomas e como usar o plano de medicação.

O registro diário de sintomas da asma e a utilização de broncodilatador auxiliaram muito o paciente e o médico no reconhecimento dos períodos das exacerbações da doença. Utilizamos um modelo simplificado de registro diário, que foi distribuído na primeira consulta e os pacientes e/ou familiares foram orientados sobre a forma correta de usá-lo. Apesar da orientação exaustiva sobre a forma correta de preenchimento, observamos que cerca de $10 \%$ dos doentes não foram capazes de preenchê-lo adequadamente na C6.

Podemos observar que, em algumas consultas, houve decréscimo no índice de acerto de alguns itens. Este fato pode ser decorrente da flutuação natural constatada durante uma transferência de conhecimento aplicada em sessões. Uma das limitações que observamos foi o tempo despendido para a transferência do conhecimento. Outra explicação para essa variação seria a real necessidade de reforço que existe no processo educativo do pacientes. Alguns trabalhos demonstraram que após um programa de educação há redução progressiva do conhecimento $e$ habilidade adquirida, refletindo a necessidade de reforço educativo constante para esses pacientes ${ }^{(25)}$.

No nosso estudo observamos que a mudança de comportamento dos pacientes em relação aos itens trabalhados variou em torno de três sessões, demonstrando o tempo prolongado para adaptação e mudança de comportamento dos pacientes. No Canadá, Turner et al. ${ }^{(29)} \mathrm{de}-$ monstraram melhora significante das medidas de desfecho logo após a $1^{\underline{a}}$ sessão educativa. Semelhante ao nosso estudo, as sessões eram mensais. Uma das possíveis explicações para essa diferença pode estar no nível socioeconômico das populações-alvo, um fator que comprovadamente interfere na mudança de comportamento e no aprendizado.

O programa de orientação individualizada tem como objetivo restabelecer o doente, a prevenção das crises e o incentivo do autocuidado(30). As intervenções propostas repercutem no dia-a-dia do indivíduo, traduzindo-se em mudanças na sua qualidade de vida. Nossa atenção deve estar voltada não somente para o quanto se vive, mas especialmente, para o como se vive. A intervenção no binômio quantidade e qualidade de vida é extremamente importante, principalmente pelo caráter crônico da doença(31).

Quanto à qualidade de vida, observamos que houve melhora significativa, especialmente nas áreas de limitação física e freqüência de sintomas. A melhora da qualidade de vida apresentada pelos pacientes reflete melhora do controle da doença, tanto do ponto de vista farmacológico como de controle ambiental. Em relação ao tratamento farmacológico, a medicação era ajustada conforme a necessidade clínica do paciente e não foi fornecido nenhum tipo de medicamento. A melhora talvez se deva ao fato da maior adesão ao tratamento e do aumento no conhecimento e habilidade da técnica correta do uso do aerossol.

A eficácia dos planos de educação em reduzir os parâmetros de morbidade e mortalidade são inquestionáveis, inclusive no nosso meio ${ }^{(11,12)}$, porém, a forma de proporcionar essa educação é diversa e não há uma análise crítica sobre o melhor programa para determinada população.

Observamos que o conhecimento dos pacientes é deficiente tanto em relação aos conceitos sobre a doença e medicação utilizada como em relação às habilidades necessárias para a utilização de medicação $e$ ações para o melhor controle da doença, como o preenchimento do 
registro diário. Isso reforça a necessidade de intervenção educativa constante e de profissionais bem treinados no manejo adequado da doença.

Relevante salientar a importância da padronização de aplicação de educação que leve a racionalização dos itens abordados, facilitando o reconhecimento das dificuldades dos pacientes, além de melhora na transferência de conhecimento pelo profissional ao paciente, e racionalização das intervenções necessárias, considerando as limitações do nosso meio e dos pacientes, orientando ações possíveis de realizar para um controle ambiental adequado, agilizando o atendimento e tornando o programa fácil de reproduzir.

O presente estudo permite-nos concluir que o modelo de pós-consulta padronizado elaborado foi aplicável dentro de uma rotina ambulatorial e possibilitou-nos avaliar o conhecimento $e$ aprendizado dos pacientes no início $e$ durante o programa. Após as intervenções realizadas, a qualidade de vida global e seus componentes - limitação física, freqüência de sintomas, adesão ao tratamento $e$ socioeconômico - melhoraram de maneira significante; não houve melhora significante apenas do componente psicossocial.

\section{REFERÊNCIAS}

1. National Asthma Education Program, Expert Painel Report. Guidelines for the diagnosis and management of asthma. Bethesda: National Institutes of Health, 1997.

2. Sullivan S, Elixhauser A, Buist S, Luce BR, Eisenberg J. National asthma education and prevention program working group report on cost effectiveness of asthma care. Am J Respir Crit Care Med 1996;154: S84-95.

3. Sociedade Brasileira de Alergia e Imunologia, Sociedade Brasileira de Pediatria e Sociedade Brasileira de Pneumologia e Tisiologia. II Consenso Brasileiro no Manejo da Asma. J Pneumol 1998;24:171-276.

4. Rea H, Scragg R, Jackson R, Beaglehole R, Fenwick J, Sutherland DC. A case-control study of deaths from asthma. Thorax 1986;41: 833-9.

5. Strunk RC, Mrazek DA, Fuhrmann GS, Labrecque JF. Physiologic and psychological characteristics associated with deaths due to asthma in childhood. A case-control study of deaths. JAMA 1985; 254:1193-8.

6. Sears MR. Changing patterns of asthma morbidity and mortality. J Invest Allergol Clin Immunol 1995;5:66-72.

7. Evans D. To help patients control asthma the clinician must be a good listener and teacher. Thorax 1993;48:685-7.

8. Mayo PH, Richman J, Harris HW. Results of a program to reduce admissions for adults asthma. Ann Intern Med 1990;112:864-71.

9. Lahdensuo A, Haahtela T, Herrala J, Kava T, Kiviranta K, Kuusisto P, et al. Randomized comparison of guided self management and traditional treatment of asthma over one year. BMJ 1996;312:748-52.

10. Choy KL, Tong M, Ko F, Li ST, Ho J, Chan R, et al. Evaluation of the efficacy of a hospital-based asthma education programme in patients of low socioeconomic status in Hong Kong. Clin Exp Allergy 1999; 29:84-90.
11. De Oliveira MA, Bruno VF, Ballini LS, Jardim JRB, Fernandes ALG. Evaluation of an educational program for asthma control in adults. $J$ Asthma 1997;34:395-403.

12. De Oliveira MA, Faresin SM, Bruno VF, Bittencourt AR, Fernandes ALG. Evaluation of an educational programme for socially deprived asthma patients. Eur Respir J 1999;14:1-7.

13. Global initiative for asthma. Global strategy for asthma and prevention NHLBI/WHO Workshop Report. Natl Heart Lung Blood Inst 1995; 95:3659

14. Sociedade Brasileira de Alergia e Imunologia. Sociedade Brasileira de Pediatria e Sociedade Brasileira de Pneumologia e Tisiologia - I Consenso Brasileiro de Educação em Asma. J Pneumol 1996;22(Supl 1): $1-24$.

15. Maiman L, Green LW, Gibson G, Mackenzie EJ. Education for self treatment by adult asthmatics. JAMA 1979;241:1919-22.

16. Charlton I, Charlton G, Broomfield J, Mullee MA. Audit of the effect of a nurse run asthma clinic on workload and patient morbidity in a general practice. Br J Gen Pract 1991;41:227-31.

17. American Thoracic Society (ATS). Standards for the diagnosis and care of patients with chronic obstructive pulmonary disease (COPD) and asthma. Am Rev Respir Dis 1987;136:225-44.

18. Fernandes ALG, Oliveira MA. Avaliação de qualidade de vida nas asma. J Pneumol 1997;23:148-52.

19. Manzella BA, Brooks CM, Richards JM,Windsor RN, Soong S, Bailey WC. Assessing the use of metered dose inhalers by adults with asthma. J Asthma 1989;26:223-30.

20. Siegel S, Castellan Jr NJ. Nonparametrics statistics second. New York: McGraw-Hill; 1988.

21. Takahashi OC, Haddad MCL, Guariente MHDM, Almeida HGG, Scarinci IC, Takahashi I. Atendimento ambulatorial e interdisciplinar ao paciente diabético. Acta Paul Enf 1993;6:43-7.

22. Stefanelli MC. Comunicação com o paciente - teoria e ensino. $2^{\underline{a}}$ ed. São Paulo: Robe Editorial, 1993; cap. 1.

23. Rebuck D, Dzyngel B, Khan K, Kesten RN, Chapman KR. The effect of structural versus conventional inhaler education in medical housestaff. J Asthma 1996;33:385-93.

24. Sandrini A, Jacomossi A, Faresin SM, Fernandes ALG, Jardim JR. Aprendizado do uso do inalador dosimetrado após explicação por pneumologista. J Pneumol 2001;27:7-10.

25. Kolbe J, Vamos M, James F, Elkind G, Garrett J. Assessment of practical knowledge of self-management of acute asthma. Chest 1996; 109:86-90.

26. Kolbe J, Vamos M, Fergusson W, Elkind G, Garrett J. Differential influences on asthma self-management knowledge and self-management behavior in acute severe asthma. Chest 1996;110:1463-8.

27. Boulet LP, Chapman KR, Green LW, Fitzgerald JM. Asthma education. Chest 1994;106:S184-95.

28. Osman L. Guided self-management and patient education in asthma. Br J Nurs 1996;5:785-9.

29. Turner MO, Taylor D, Bennett R, Fitzgerald MJ. A randomized trial comparing peak expiratory flow and symptom self-management plans for patients with asthma attending a primary care clinic. Am J Respir Crit Care Med 1998;157:540-6.

30. Dupas G, Pinto IC, Mendes MD, Benedini Z. Reflexão e síntese acerca do modelo do auto-cuidado de Orem. Acta Paul Enf 1994;7:19-26.

31. Lima LS, Coiado CRP, Souza CB, Jamnik S, Bogossian M. Qualidade de vida em portadores de bronquiectasia antes e após programa de educação em saúde. J Pneumol 1996;22(Suppl):7A. 


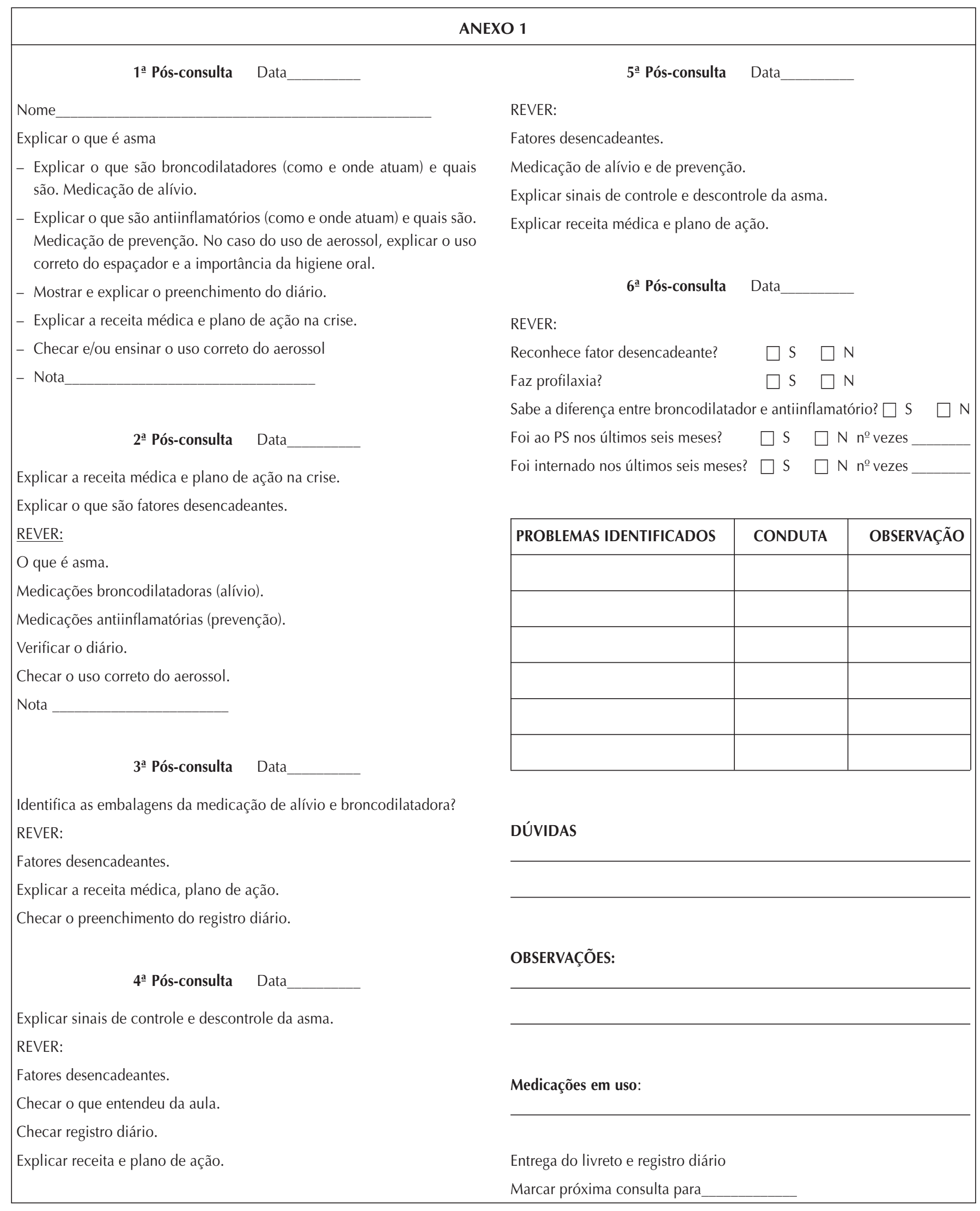

